\title{
literature report
}

\section{Coupling adhesion to actin bundles in theinner ear}

\section{Novel functions for novel cadherins}

\author{
Astrid Kraemer ${ }^{1} \&$ Alpha S. Yap ${ }^{1,2+}$
}

Genotype and phenotype define two poles of modern biology. The great challenge is to understand the connections that translate gene products into tissue architecture and function. Two recent studies (Boeda et al., 2002; Siemens et al., 2002) exemplify how these connections can be identified during the development of the inner ear.

The sensory hair cells of the inner ear detect sound and postural change by sensing movement in the endolymph fluid of the cochlea and the semicircular canals, respectively. At the subcellular level, mechano-sensing is mediated by organelles called stereocilia (SC) - fine, finger-like protrusions that project from the apices of the hair cells. The morphology and organization of SC are strikingly precise: in mature hair cells, SC increase regularly in height from one side of the cell to the other and are attached to one another by several lateral connections, thereby integrating individual SC into functional units. Developmental mechanisms must therefore exist to coordinate the growth and lateral interactions between SC.

The importance of SC morphogenesis becomes apparent in U sher syndrome type 1 . Mouse models of this disease show deafness and vestibular dysfunction and have SC that grow haphazardly and lose lateral interactions with one another. Seven genetic loci are independently able to cause this syndrome when mutated (reviewed in Petit, 2001). The corresponding genes for four of these loci encode the unconventional motor protein myosin VIla, the cytoplasmic protein harmonin, and two transmembrane proteins of the cadherin superfamily, cadherin $23(\mathrm{CDH} 23)$ and protocadherin 15. How these apparently diverse gene products generate a common phenotype was unclear. However, the two new studies highlighted here have provided a significant advance by demonstrating biochemical and morphological interactions that involve the first three of these proteins.

The cadherins are a superfamily of cell-surface glycoproteins that mediate cell-cell adhesion (Yagi \& Takeichi, 2000). CDH 23 (Di Palma et al., 2001) is a novel cadherin that is distinguished by a very large extracellular domain that most resembles the D rosophila Fat protein and by a cytoplasmic domain that lacks any identifiable

\footnotetext{
11nstitutefor M olecular Bioscienceand ${ }^{2}$ School for Biomedical Science, The University of Queensland, St Lucia, Brisbane, Queensland 4072, Australia

+Corresponding author. Tel: +61 73365 4906; Fax: +61 73365 1766;

E-mail: a.yap@mailbox.uq.edu.au

EM BO reports 4 (2003)

doi:10.1038/s.embor.embor 775

Submitted 27 December 2002; accepted 22 January 2003 Published online 14 February 2003
}

features except for two putative PDZ-binding interfaces (PBIs). In this regard, $\mathrm{CDH} 23$ is distinct from the better-characterized classical cadherins (such as $\mathrm{E}$ - and $\mathrm{N}$-cadherin), the conserved cytoplasmic tails of which interact with catenin proteins, and thus link them to the actin cytoskeleton. Notably, harmonin proteins contain several PDZ domains and, in the recent papers, both research groups use yeast two-hybrid assays and purified proteins to show that $\mathrm{CDH} 23$ and harmonin interact directly (Boeda et al., 2002; Siemens et al., 2002). This interaction seems to involve the PDZ2 domain of harmonin and the carboxy-terminal PBI of CD H23 (Siemens et al., 2002), thus defining a new mode of cytoplasmic interaction for cadherins.

Several harmonin transcripts exist in the inner ear, of which the harmonin $b$ transcript seems to be specific for this organ. Importantly, Boeda et al. (2002) further identify a close spatio-temporal relationship between $\mathrm{CDH} 23$ and harmonin $\mathrm{b}$ in developing hair cells. Both are first detectable in the earliest apical protrusions that extend to become SC. These proteins completely co-localize-initially being found throughout the nascent SC, but becoming progressively restricted to the apices of the hair cells as the SC elongate. They eventually disappear in mature hair cells. Boeda et al. (2002) also show that harmonin PDZ1 interacts with myosin VIla. Strikingly, in mice
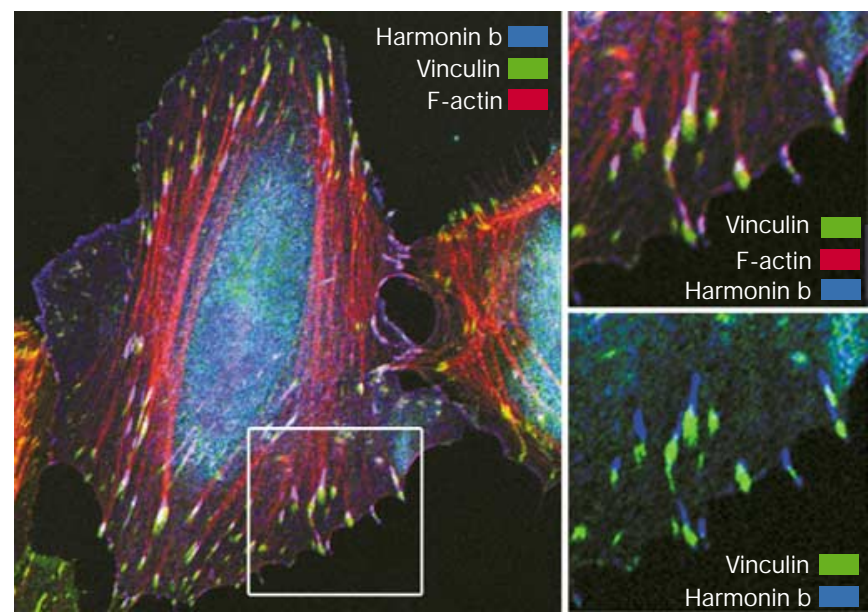

Fig. 1 | HeLa cell expressing harmonin b. Six hours after transfection, harmonin $b$ (blue) is enriched at theextremities of actin stress fibres (red), closeto the focal adhesion plaques visualized by anti-vinculin staining ( $g r e e n)$. The righthand panels arehigher magnification views of the boxed area. The figurewas kindly provided by B. Boeda and A. El-Amraoui (Institut Pasteur, France). 


\section{reviews}

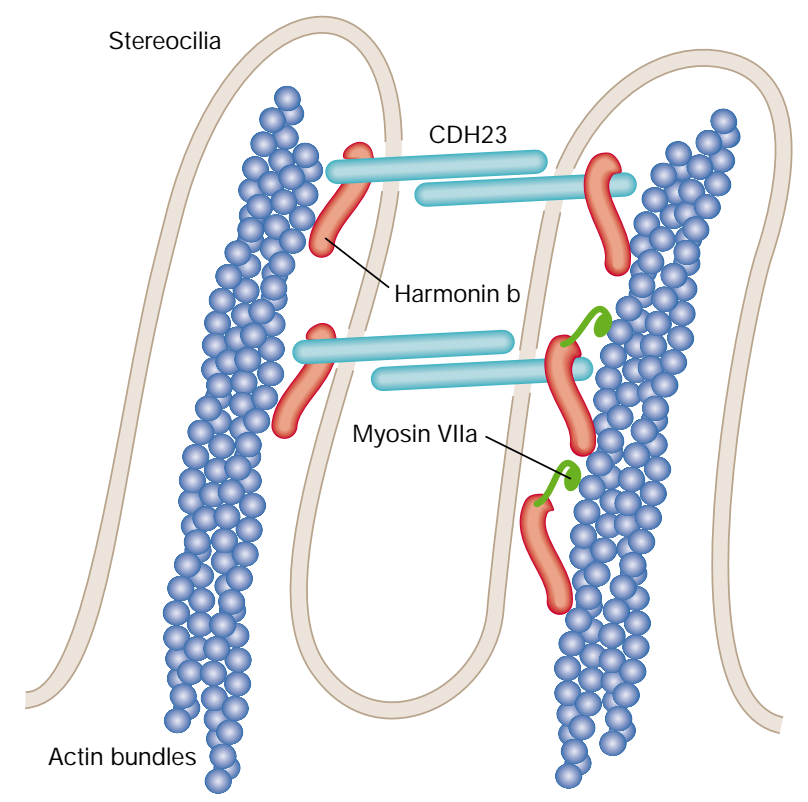

Fig. 2 Schematic model for the biochemical and functional interactions between cadherin 23 ( $C D H 23$ ), harmonin b and myosin VIIa. Binding of CDH 23 to harmonin b couples adhesiveinteractions between growing stereocilia (SC) to thecoreactin bundles that arenecessary for stereocilial growth. This would allow growth of neighbouring SC to be precisely coordinated. An association between harmonin b and myosin VII a may transport harmonin b to thecadherin, as suggested by Boeda et al. (2002). Alternatively, a ternary complex of myosin VIIa, $\mathrm{CDH} 23$ and harmonin b, might participatein thecadherin-actin interaction supported by harmonin $b$.

that are homozygous for a myosin VIla mutation, harmonin $b$ failed to co-accumulate with $\mathrm{CDH} 23$. This caused the SC to grow abnormally- varying irregularly in height and splaying out from one another - as if their lateral interactions were lost. Together, these data strongly suggest that functional as well as biochemical interactions involving myosin VIla, CDH23 and harmonin b are somehow necessary for proper SC morphogenesis. N ote, however, that although mapping of individual binding interactions suggests that a ternary complex of $\mathrm{CDH} 23$, harmonin $\mathrm{b}$ and myosin VIla is possible, this has yet to be tested experimentally.

How, then, might $\mathrm{CDH} 23$ and harmonin b support SC development? An exciting clue is the demonstration that harmonin $b$ can bundle actin filaments, both in vitro and when overexpressed in fibroblasts (Boeda et al., 2002; Fig. 1). It is important to remember that SC are tightly packed with actin filaments that are linked laterally into bundles. Similar to other actin-rich cell-surface protrusions, SC are believed to grow both by polymerization of individual actin filaments and by the incorporation of these filaments into bundles (DeRosier \& Tilney, 2000). Filament bundling by harmonin b would thus be predicted to promote SC growth during morphogenesis. Moreover, adhesion mediated by $\mathrm{CDH} 23$ could couple nascent SC to one another, ensuring lateral association between $\mathrm{SC}$ as they grow. Consistent with this, $\mathrm{CDH} 23$ could be localized to lateral tip-to-tip contacts in nascent SC by immuno-electron microscopy (Boeda et al., 2002). Thus, a membrane-based complex of $\mathrm{CDH} 23$ and harmonin b provides a mechanism both to promote actin filament bundling (individual SC growth) and to initiate the lateral associations between SC that couple them into functional units as they grow (Fig. 2). Notably, in this model, $\mathrm{CDH} 23$ would mediate cis-interactions between membrane projections of the same cells, a novel effect that contrasts with the trans-interactions mediated by classical cadherins.

Of course, these data open avenues for further experiments. The ability of cadherin superfamily proteins to mediate adhesion has been proven principally for the classical cadherins, but $\mathrm{CDH} 23$ differs markedly from these proteins in primary sequence. Therefore, first it will be important to test directly whether $\mathrm{CDH} 23$ is a true adhesion molecule, and if so, whether harmonin b can itself affect the adhesive activity of $\mathrm{CDH} 23$. Interestingly, harmonins can oligomerize (Siemens et al., 2002); could this support lateral clustering of CD 23 to modulate adhesion as occurs with classical cadherins (Yap et al., 1998)? Second, the precise role of myosin VIla remains to be elucidated. Boeda et al. (2002) suggest that this motor protein may transport harmonin $\mathrm{b}$ in SC; it will be interesting to determine if myosin VIla can influence the $\mathrm{CDH} 23$-harmonin complex itself. Additionally, if myosin VIla could form a ternary complex with $\mathrm{CDH} 23$ and harmonin b, it could potentially stabilize or exert force on the membrane-actin interaction mediated by the latter proteins. Also, does the $\mathrm{CDH} 23-$ harmonin b complex exert a morphogenetic effect on actin dynamics in SC? Is the complex passively anchored on actin bundles, or can it determine the sites where bundling occurs in developing SC? If so, this would add to the increasing evidence that cadherins can exert active, instructive effects on the actin cytoskeleton (Kovacs et al., 2002). Although there is much to be learned, these recent papers provide important insights into the interactions between gene products that generate a functional organelle.

\section{REFERENCES}

Boeda, B. et al. (2002) M yosin VIla, harmonin and cadherin 23, three U sher I gene products that cooperate to shape the sensory hair cell bundle. EMBO J., 21, 6689-6699.

DeRosier, D.J. \& Tilney, L.G . (2000) F-actin bundles are derivatives of microvilli: What does this tell us about how bundles might form? J. Cell Biol., 148, 1-6. Di Palma, F., Holme, R.H., Bryda, E.C., Belyantseva, I.A., Pellegrino, R., Kachar, B., Steel, K.P. \& N oben-Trauth, K. (2001) Mutations in Cdh23, encoding a new type of cadherin, cause stereocilia disorganization in waltzer, the mouse model for U sher syndrome type 1D. Nature Genet, 27, 103-107.

Kovacs, E.M., Goodwin, M., Ali, R.G., Paterson, A.D. \& Yap, A.S. (2002) Cadherin-directed actin assembly: E-cadherin physically associates with the Arp2/3 complex to direct actin assembly in nascent adhesive contacts. Curr. Biol., 12, 379-382.

Petit, C. (2001) U sher syndrome: from genetics to pathogenesis. Annu. Rev. Genomics H um. Genet., 2, 271-297.

Siemens, J., Kazmierczak, P., Reynolds, A., Sticker, M., Littlewood-Evans, A. \& Muller, U. (2002) The U sher syndrome proteins cadherin 23 and harmonin form a complex by means of PDZ-domain interactions. Proc. Natl Acad. Sci. USA, 99, 14946-14951.

Yagi, T. \& Takeichi, M. (2000) Cadherin superfamily genes: functions, genomic organization, and neurologic diversity. Genes Dev., 14, 1169-1180.

Yap, A.S., N iessen, C. \& G umbiner, B.M . (1998) The juxtamembrane region of the cadherin cytoplasmic tail supports lateral clustering, adhesive strengthening and interaction with p120 ctr. J. Cell Biol., 141, 779-789.
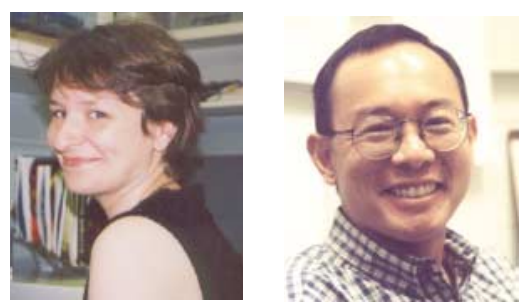

Astrid Kraemer
Alpha S. Yap 